\title{
Crystal structures of oseltamivir-resistant influenza virus neuraminidase mutants
}

\author{
Patrick J. Collins ${ }^{1}$, Lesley F. Haire ${ }^{1}$, Yi Pu Lin ${ }^{1}$, Junfeng Liu ${ }^{1}$, Rupert J. Russell ${ }^{2}$, Philip A. Walker ${ }^{1}$, John J. Skehel ${ }^{1}$, \\ Stephen R. Martin ${ }^{1}$, Alan J. Hay ${ }^{1} \&$ Steven J. Gamblin ${ }^{1}$
}

The potential impact of pandemic influenza makes effective measures to limit the spread and morbidity of virus infection a public health priority. Antiviral drugs are seen as essential requirements for control of initial influenza outbreaks caused by a new virus, and in pre-pandemic plans there is a heavy reliance on drug stockpiles. The principal target for these drugs is a virus surface glycoprotein, neuraminidase, which facilitates the release of nascent virus and thus the spread of infection. Oseltamivir (Tamiflu) and zanamivir (Relenza) are two currently used neuraminidase inhibitors that were developed using knowledge of the enzyme structure $^{1,2}$. It has been proposed that the closer such inhibitors resemble the natural substrate, the less likely they are to select drug-resistant mutant viruses that retain viability ${ }^{3}$. However, there have been reports of drug-resistant mutant selection in vitro ${ }^{4}$ and from infected humans ${ }^{5,6}$. We report here the enzymatic properties and crystal structures of neuraminidase mutants from H5N1-infected patients that explain the molecular basis of resistance. Our results show that these mutants are resistant to oseltamivir but still strongly inhibited by zanamivir owing to an altered hydrophobic pocket in the active site of the enzyme required for oseltamivir binding. Together with recent reports of the viability and pathogenesis of $\mathrm{H} 5 \mathrm{~N} 1$ (ref. 7) and $\mathrm{H} 1 \mathrm{~N} 1$ (ref. 8) viruses with neuraminidases carrying these mutations, our results indicate that it would be prudent for pandemic stockpiles of oseltamivir to be augmented by additional antiviral drugs, including zanamivir.

Influenza neuraminidase (NA) functions in virus infection to remove sialic acid from cell-surface receptors so that newly made viruses are released and able to spread to uninfected cells 9 . To inhibit virus propagation NA inhibitors were developed, informed in part by the crystal structures of several NAs belonging to one of the two genetically defined NA groups of influenza $\mathrm{A}^{10,11}$, but with the objective that they should be active against all influenza viruses ${ }^{12}$. The outcome of this work includes the licensing of the drugs oseltamivir $(\text { Tamiflu) })^{1}$ and zanamivir (Relenza) ${ }^{2}$, which are active against NAs from group 1 and 2 influenza A as well as influenza B viruses ${ }^{5}$. From this work it was proposed that inhibitors that closely resemble the natural sialic acid substrate of NA are unlikely to select drug-resistant mutants that retain normal enzyme activity ${ }^{3}$. Thus, viruses that are resistant to such drugs ought to be less viable ${ }^{13-15}$. In the present study we have determined binding and inhibitory parameters for oseltamivir and zanamivir interacting with wild-type and three mutant $\mathrm{H} 5 \mathrm{~N} 1$ neuraminidases. The mutations involved are: (1) His274Tyr, the principal mutation isolated in association with oseltamivir treatment that is specific to the $\mathrm{N} 1$ group $^{16}$ and that has recently been shown to be present in substantial numbers of H1N1 viruses isolated from humans $^{8}$; (2) Asn294Ser, which has been identified in viruses containing either N1 or N2 NAs, isolated from patients treated with oseltamivir $^{17,18}$; and (3) a variant of N1 in which the generally conserved tyrosine residue at position 252 was replaced by histidine. This substitution was not associated with drug treatment and was found only in one clade of H5N1 viruses isolated from infected humans in Vietnam and Cambodia in 2004 and 2005 (ref. 19).

We used a fluorescent substrate (see Methods and Supplementary Information) to measure enzyme activity $\left(V_{\mathrm{m}}\right)$ and Michaelis constant $\left(K_{\mathrm{m}}\right)$ as well as inhibitory constants $\left(K_{\mathrm{I}}\right)$ for the different NAs. The values for enzyme activity $\left(V_{\mathrm{m}}\right)$ given in Table 1 for wild-type and mutant NAs are similar, and there is at most a ninefold difference in the $K_{\mathrm{m}}$ values. These results are similar to those previously reported ${ }^{20,21}$ and support the notion that substitution of amino acids adjacent to the active site, that do not interact with the substrate, can be accommodated without significantly affecting NA activity ${ }^{22}$. Inspection of the inhibitory constants $\left(K_{\mathrm{I}}\right)$ in Table 1 shows that none of the mutations has a major impact on the effectiveness of zanamivir. However, two of them substantially reduce the inhibition caused by oseltamivir: the His 274 Tyr substitution by a factor of 265 and the Asn294Ser by a factor of 81 . In contrast, the Tyr 252 His substitution leads to a tenfold increase in sensitivity to this inhibitor.

We compared the kinetics of oseltamivir and zanamivir binding to wild-type NA (see Methods) to show that the approach to equilibrium is approximately three times faster with oseltamivir and to

Table $1 \mid$ Activity, binding and kinetic parameters for N1 neuraminidases

\begin{tabular}{|c|c|c|c|c|c|c|c|c|}
\hline NA type & $\begin{array}{l}\quad V_{\mathrm{m}} \\
\text { relative to wild type }\end{array}$ & $\begin{array}{c}K_{m} \\
(\mu M)\end{array}$ & $\begin{array}{l}\text { Oseltamivir } \\
\text { relative } K_{1}^{*}\end{array}$ & $\begin{array}{l}\text { Zanamivir } \\
\text { relative } K_{\|} \dagger\end{array}$ & $\begin{array}{c}k_{\text {on }}\left(\mu \mathrm{M}^{-1} \mathrm{~s}^{-1}\right) \\
\text { oseltamivir }\end{array}$ & $\begin{array}{c}k_{\text {off }}\left(\mathrm{s}^{-1}\right) \\
\text { oseltamivir }\left(\times 10^{4}\right)\end{array}$ & $\begin{array}{c}k_{\text {on }}\left(\mu \mathrm{M}^{-1} \mathrm{~s}^{-1}\right) \\
\text { zanamivir }\end{array}$ & $\begin{array}{c}k_{\text {off }}\left(\mathrm{s}^{-1}\right) \\
\text { zanamivir }\left(\times 10^{4}\right)\end{array}$ \\
\hline Wild type & 1.0 & 6.3 & 1.0 & 1.0 & $2.52(0.21)$ & $8.1(1.2)$ & $0.95(0.08)$ & $0.95(0.13)$ \\
\hline His274Tyr & 0.8 & 27.0 & 265 & 1.9 & $0.24(0.06)$ & $180(30) \div$ & $0.35(0.02)$ & $0.67(0.08)$ \\
\hline Asn294Ser & 1.15 & 53.0 & 81 & 7.2 & $1.1(0.18)$ & $235(40) \dagger$ & $0.52(0.04)$ & $3.7(0.6)$ \\
\hline Tyr252His & 0.94 & 7.5 & 0.1 & 1.2 & $3.9(0.15)$ & $1.25(0.13)$ & $1.38(0.15)$ & $1.66(0.33)$ \\
\hline
\end{tabular}

$K_{\mathrm{m}}$ values are from three determinations; $K_{1}$ values from at least six measurements. Values in parentheses represent the standard deviations obtained from linear least squares fits to $k_{\text {obs }}$ values as a function of substrate and inhibitor concentrations, as shown in Supplementary Information. $k_{\mathrm{on}}$ and $k_{\mathrm{off}}$ are the association and dissociation rate constants, respectively.

* Oseltamivir relative $K_{1}$ is $K_{1}$ (mutant) $/ K_{1}$ (wild type), where wild type $=0.32 \mathrm{nM}$.

$\dagger$ Zanamivir relative $K_{1}$ is $K_{1}$ (mutant) $/ K_{1}$ (wild-type), where wild type $=0.1 \mathrm{nM}$

t. Directly determined.

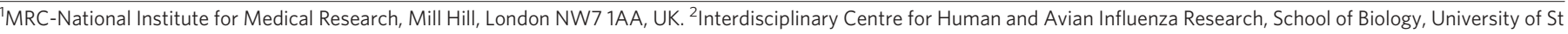
Andrews, Fife KY16 9ST, UK. 
confirm that zanamivir is the slightly more potent inhibitor (Fig. 1a, $\mathrm{b}$ and Table 1). Our kinetic data also show that between the wild-type and mutant enzymes there are, as expected, much greater differences for the rates of association and dissociation with oseltamivir than with zanamivir. The reduced affinity of the His274Tyr mutant NA for oseltamivir arises from a tenfold poorer association rate constant and a 25-fold enhanced dissociation rate constant. For the Asn294Ser mutant the reduced affinity is largely accounted for by a 35-fold increase in off-rate for the inhibitor whereas the Tyr252His variant is more sensitive to oseltamivir mostly because of a 6.5 -fold decrease in the off-rate. Notably, there is close agreement between the inhibitory constants and the ratio of association and dissociation rates
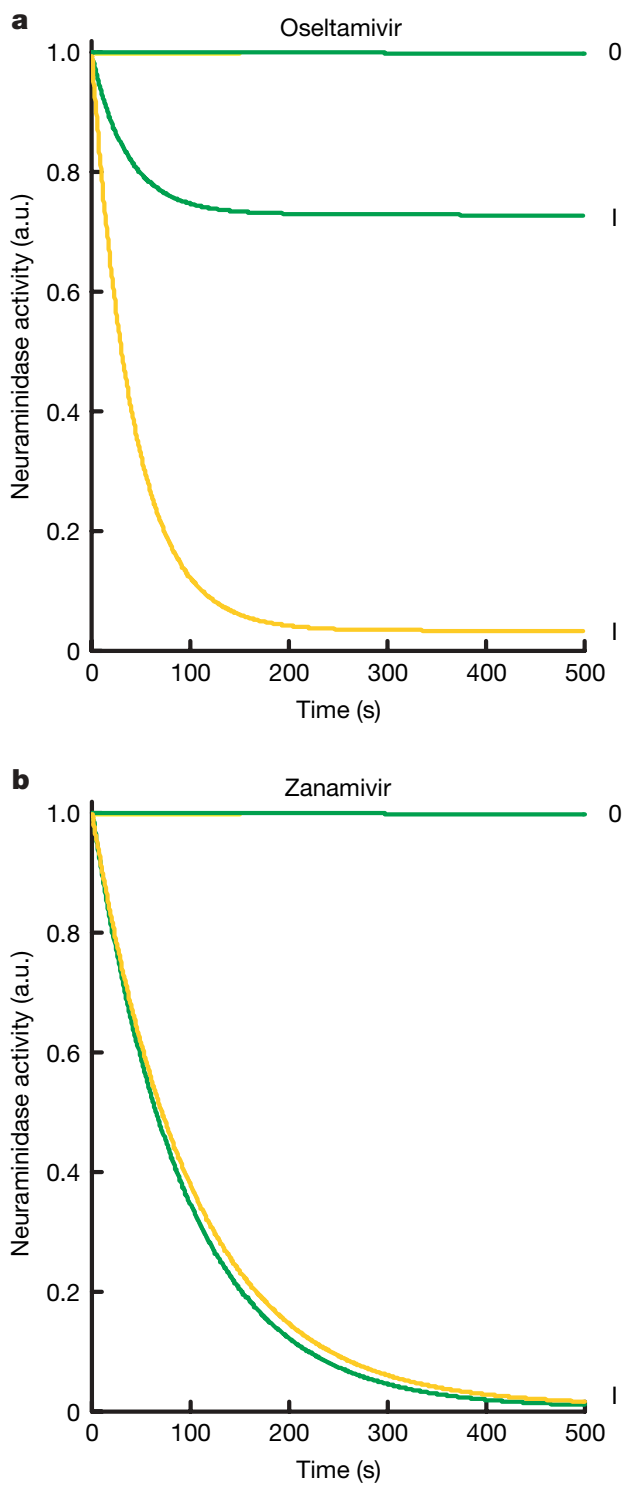

Figure 1 | Neuraminidase activity monitored using a fluorescent assay. NA activity for wild type (yellow) and His274Tyr (green) mutant proteins in the absence (labelled 0) and presence (labelled I) of $85 \mathrm{nM}$ inhibitor at $50 \mu \mathrm{M}$ substrate. a, b, Effect of oseltamivir (a) and zanamivir (b). For the His274Tyr mutant the approach to equilibrium occurs at a similar rate for the two inhibitors even though oseltamivir is the much poorer inhibitor. At the oseltamivir concentration used the reduced contribution of the on-rate constant to the observed rate is almost exactly compensated for by the increased contribution from the off-rate constant. Although oseltamivir has sometimes been referred to as a slow-binding inhibitor ${ }^{21,30}$, the association rate constants that we determined are within the range frequently observed for the interaction of small molecules with proteins. It is the use both here and elsewhere of very low inhibitor concentrations that result in a slow approach to equilibrium, not the kinetics of binding. measured kinetically, giving us confidence in the robustness of the data.

We used X-ray crystallography to understand the structural basis for the poorer binding of oseltamivir to the His274Tyr and Asn294Ser mutant NAs relative to wild type (Fig. 2a, b). Crystals of the His274Tyr mutant NA in complex with both inhibitors diffracted to high resolution and produced clearly defined electron density for the bound oseltamivir (Fig. 2c) and zanamivir (Fig. 2d). Relevant crystallographic statistics are given in Supplementary Table 1. With respect to binding to wild-type NA the key difference between oseltamivir and zanamivir is that oseltamivir has a hydrophobic pentyloxy substituent at the C6 position rather than a polar glycerol group in zanamivir (as is also the case for the sialic acid substrate, Fig. 2b). Binding of oseltamivir to wild-type NA involves a conformational change in the side chain of Glu 276 relative to the ligandfree enzyme $e^{1,3,23}$. In its new conformation, the carboxyl group of Glu 276 is oriented away from the hydrophobic pentyloxy group of oseltamivir, the latter making hydrophobic contact with the $C \beta$ methylene of Glu 276 (Fig. 2c, yellow coloured chain). Binding of zanamivir, like sialic acid ${ }^{24}$, involves hydrogen-bond formation between the carboxyl group of Glu 276 and the 8- and 9-hydroxyl groups of the glycerol moiety of the inhibitor and requires no change in side-chain conformation (Fig. 2d, yellow coloured chain). The structure of the His274Tyr-oseltamivir complex (Fig. 2c) shows that substitution by the bulkier tyrosine residue pushes the carboxyl group of Glu $2762 \AA$ farther into the binding site. In this position the charged group disrupts the otherwise hydrophobic pocket that normally accommodates the pentyloxy substituent of oseltamivir and causes a change in the conformation of the inhibitor such that its C9 and C91 carbons move about $2.5 \AA$ from their wild-type NAbound position (Fig. 2c). This result is similar to the observations on inhibitor binding to the Arg292Lys mutation of N9 (ref. 3). In contrast, the structure of the His274Tyr-zanamivir complex (Fig. 2d) shows how the tyrosine residue is accommodated by a small movement in the side chain of Glu 276. Moreover, the adjustment is made without disrupting the hydrogen bonds made between zanamivir and Glu 276 in wild-type NA.

The two structures thus provide a direct explanation for the reduction in the binding affinity of the His274Tyr mutant for oseltamivir without significantly altering the binding of zanamivir. The structures also confirm our earlier suggestion that the substitution of tyrosine for histidine at position 274 would not be accommodated in group 1 (N1, N4, N5 and N8) NAs without disrupting the oseltamivir-binding site, because of the presence of a bulky and conserved tyrosine at position 252 beneath residue 274 (Fig. 2a). In contrast, group 2 NAs (N2, N3, N6, N7 and N9) have the smaller threonine residue at position 252 and can accommodate the His274Tyr substitution without changing the oseltamivir-binding site ${ }^{25}$. Our binding and structural studies also provide support for the notion that zanamivir, having the same glycerol moiety at C6 as sialic acid (Fig. 2b), is less likely to encounter mutations in this region of the active site that weaken its binding without also weakening the binding of the natural substrate.

In N1 NAs residue 252 is generally conserved as tyrosine. However, in a number of $\mathrm{H} 5 \mathrm{~N} 1$ viruses isolated from humans in Vietnam in 2004-05 histidine was present at position 252 (ref. 19). As our thermodynamic and kinetics data show, this substitution results in an enzyme about 10 times more sensitive to oseltamivir but essentially unchanged with respect to zanamivir binding. We suspect that we have been unable to crystallize this Tyr252His mutant because of a more flexible structure arising from the loss of hydrogen bonds of the tyrosine side chain with both main-chain groups and the side chain of His 274. Our data indicating that the Tyr252His mutation affects oseltamivir but not zanamivir binding suggests that it is the loss of the Tyr 252 to His 274 hydrogen bond that favours the re-orientation of Glu 276 required for oseltamivir binding, thus accounting for the 
tenfold enhancement of oseltamivir binding by this mutant NA without altering its affinity for zanamivir.

We have also determined the structure of the complex of Asn294Ser with oseltamivir (Fig. 2e). This shows smaller changes in the position of the mutant-bound oseltamivir and adjacent side chains, relative to wild-type, than those seen for oseltamivir bound to the His274Tyr mutant (Fig. 2c, f). The Ser 294 side chain in the mutant is oriented so that its polar hydroxyl group forms a hydrogen bond with the carboxylate of Glu 276. By contrast, the side chain of Asn 294 in the wild-type NA is directed in approximately the opposite direction, towards Tyr 347. The loss of the asparagine side chain at position 294 enables the main-chain carbonyl of Tyr 347 to flip out, from its position in the wild-type, so that it no longer coordinates to the bound calcium ion (blue sphere in Fig. 2e). These changes lead to a less well-ordered conformation for the side chain of Tyr 347 and, presumably, a weaker hydrogen bond interaction with the carboxylate of oseltamivir (and by inference with the equivalent carboxylate in zanamivir and sialic acid). This interpretation of the Asn294Ser-oseltamivir structure is consistent with the observation (Table 1) that this mutant has an approximately sevenfold weaker binding for zanamivir (and about an eightfold higher $K_{\mathrm{m}}$ for its substrate). We suspect that the 81 -fold weaker binding of this mutant to oseltamivir results from a combination of the effect on Tyr 347 with the effect of the substituted serine residue hydrogen bonding to the side chain of Glu 276. The hydrogen bond stabilizes the serine side-chain conformation so that the polar hydroxyl group is brought into what is otherwise a hydrophobic binding site for oseltamivir in wild-type NA. Such a perturbation of the hydrophobic make-up of the binding site, which would be expected to affect the binding of oseltamivir but not zanamivir, is consistent with our a

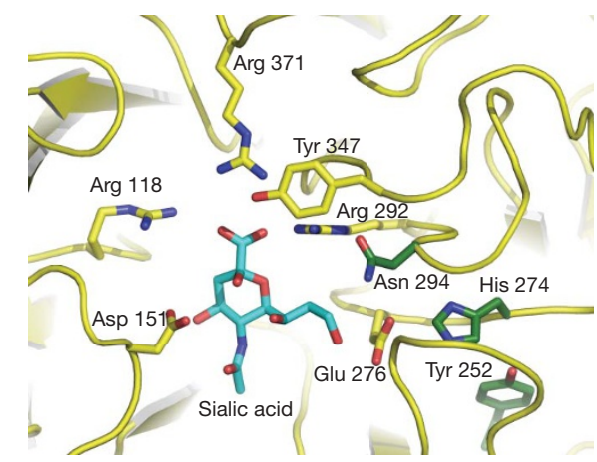

c

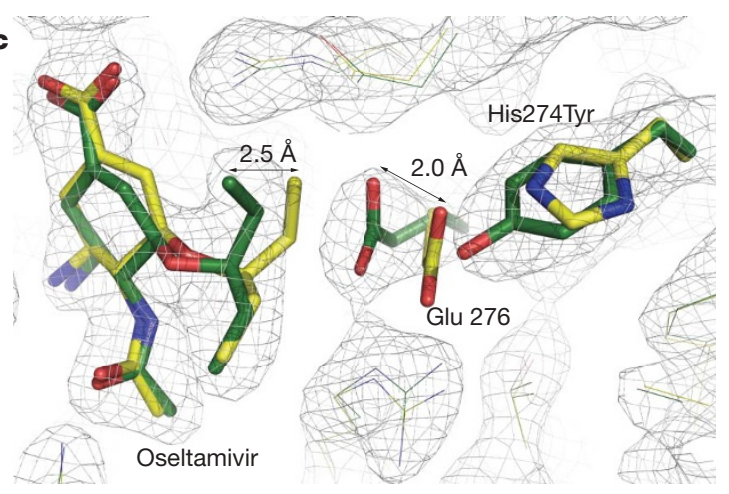

e

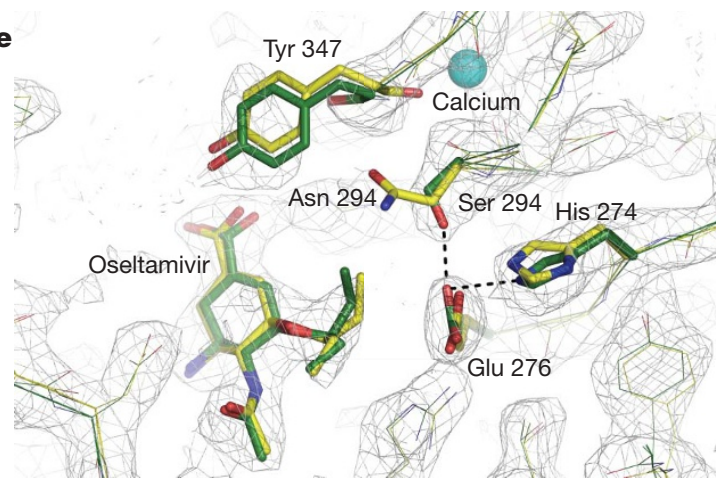

Figure 2 Structure of $\mathbf{N} 1$ neuraminidase complexes. a, Sialic acid (coloured blue) docked into the active site of wild-type N1 NA (ribbons coloured yellow) from superposition of the sialic acid complex of N2 (ref. 24) (Protein Data Bank code 2BAT). The positions of some key binding residues are shown with carbons coloured yellow, nitrogens blue and oxygens in red. The side chains of the three mutants examined in this work are shown with their carbons coloured in green (shown as the wild-type residues). $\mathbf{b}$, The structures of sialic acid (carbons coloured blue), zanamivir (carbons coloured grey) and oseltamivir (carbons coloured yellow) are shown in similar orientations with selected carbon atoms numbered. c-e, The overlaid structures of the active sites of wild-type (yellow) and mutant N1 NAs b
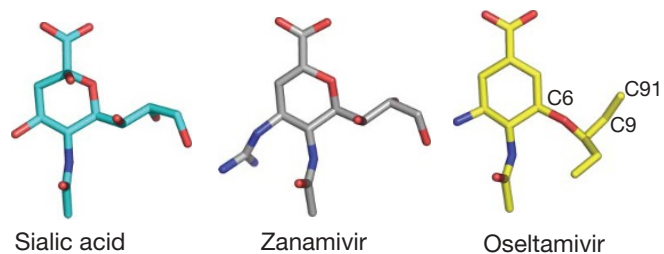

Oseltamivir

d

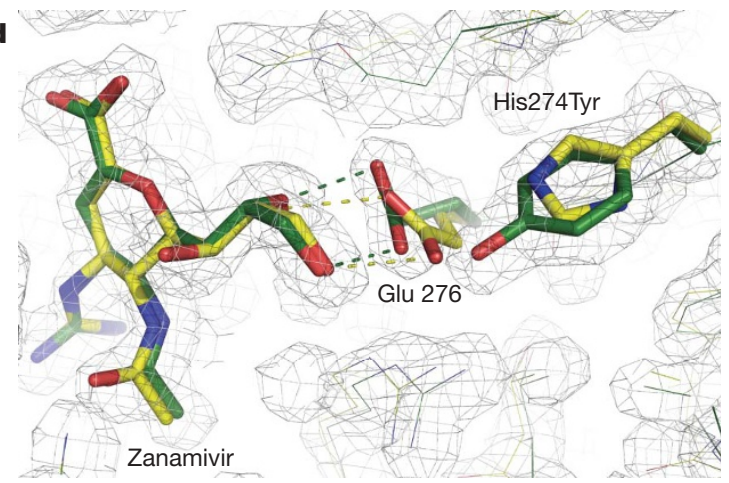

$\mathbf{f}$

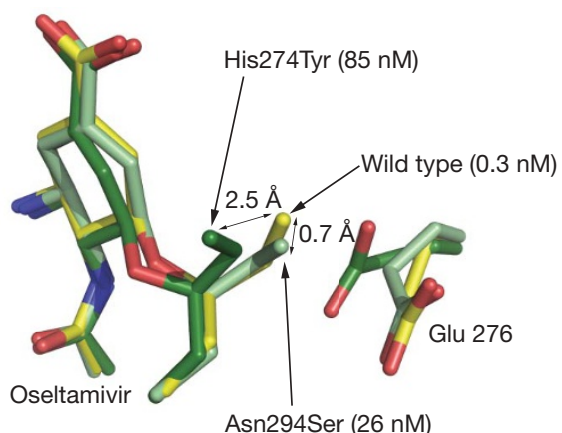

(green) are shown with bound inhibitors coloured similarly; relevant portions of electron density maps are also shown. c, His274Tyr in complex with oseltamivir. d, His274Tyr in complex with zanamivir. e, Asn294Ser in complex with oseltamivir. Dashed lines indicate selected hydrogen bonds. Electron density maps were calculated from $2 F_{\mathrm{o}}-F_{\mathrm{c}}, \alpha_{\text {calc }}$ coefficients and are contoured at $1.2 \sigma$. f, The conformation of oseltamivir and Glu 276 from three complexes is shown after superposition using protein atoms only; the carbon atoms of the inhibitor from the wild-type complex are coloured yellow, the His274Tyr in dark green and the Asn294Ser in light green. The affinities $\left(K_{\mathrm{I}}\right)$ of oseltamivir for the three NAs are given in parentheses. 
measurements of the relative affinities of this mutant for the two inhibitors.

There are currently two groups of anti-influenza drugs that target either the M2 proton channel or NA. Mutations in M2 have caused resistance among both $\mathrm{H} 3 \mathrm{~N} 2$ and $\mathrm{H} 1 \mathrm{~N} 1$ current seasonal influenza viruses and a large proportion of the avian $\mathrm{H} 5 \mathrm{~N} 1$ viruses to the drugs amantadine and rimantadine ${ }^{26}$, which block the channel, placing extra dependence on the NA-inhibitor drugs oseltamivir and zanamivir. Of these, oseltamivir has been used more frequently than zanamivir but there is little information for either drug on the rate of emergence of resistant variants in the case of the H5N1 viruses. Nevertheless, mutant viruses have been isolated from fatal H5N1 infections and among these the His274Tyr mutation described here is prominent ${ }^{6}$. Our results explain the structural basis for the resistance of this mutant to oseltamivir and for its sensitivity to zanamivir. While our work was in progress, the biological properties of $\mathrm{H} 5 \mathrm{~N} 1$ viruses carrying the His274Tyr mutation were described from studies of infected mice 7 . Previously, in ferrets, both wild-type and mutant viruses were viable, with the mutant producing only about ten times less virus than the wild-type at the peak of infection ${ }^{18}$. In the more recent studies of infected mice, both viability and pathogenicity were also closely similar for wild-type and mutant viruses ${ }^{7}$. In contrast with previous reports ${ }^{13,14}$, the viability of viruses containing the His274Tyr mutant NA has also been demonstrated recently by the isolation of numerous oseltamivir-resistant $\mathrm{H} 1 \mathrm{~N} 1$ viruses from outbreaks of human infections in Europe ${ }^{8}$. Like the mutant NAs detailed here, these enzymes are both resistant to oseltamivir and sensitive to zanamivir even though the patients from whom the mutant viruses were isolated had no known exposure to oseltamivir. Evidently, if the His274Tyr mutation also becomes common in avian H5N1, the effectiveness of oseltamivir would be limited and the value of stockpiles compromised. Considered together with the success of HAART (highly active antiretroviral therapy) against $\mathrm{HIV}^{27,28}$, it would be prudent to re-assess the suitability of single-drug, pre-pandemic stockpiles and to develop effective drug combination treatments.

\section{METHODS SUMMARY}

Site-directed mutagenesis was performed on the N1 neuraminidase gene of A/Vietnam/1203/04 (H5N1). Mutant N1 neuraminidase (NA) was prepared from a WSN-NA (H1N1) recombinant virus containing seven genes from WSN and the mutated N1 neuraminidase gene of H5N1. Recombinant viruses were grown in hens' eggs and the neuraminidase was released from the virus by bromelain digestion, and further purified, as previously described ${ }^{29}$. Purified NA concentrations were determined from their absorption spectra and enzymatic activity was measured using the fluorescent substrate $2^{\prime}$ - (4-methylumbelliferyl)$\alpha$-D- $N$-acetylneuraminic acid (MUNANA) using a JASCO FP-6300 fluorimeter with excitation and emission wavelengths of 365 and $450 \mathrm{~nm}$. NA activity changes in the presence of inhibitors were monitored as the first derivative of the fluorescence change. Crystals were obtained by vapour diffusion using 15\% PEG 3350 as precipitant. Diffraction data were collected at $100 \mathrm{~K}$ and processed using Denzo and Scalepack. The structures were solved by molecular replacement and refined using Refmac5 or PHENIX with manual model building using O or Coot.

Full Methods and any associated references are available in the online version of the paper at www.nature.com/nature.

\section{Received 14 February; accepted 1 April 2008.}

Published online 14 May 2008.

1. Kim, C. U. et al. Influenza neuraminidase inhibitors possessing a novel hydrophobic interaction in the enzyme active site: design, synthesis, and structural analysis of carbocyclic sialic acid analogues with potent anti-influenza activity. J. Am. Chem. Soc. 119, 681-690 (1997).

2. von Itzstein, M. et al. Rational design of potent sialidase-based inhibitors of influenza virus replication. Nature 363, 418-423 (1993)

3. Varghese, J. N. et al. Drug design against a shifting target: a structural basis for resistance to inhibitors in a variant of influenza virus neuraminidase. Structure 6 , 735-746 (1998).

4. McKimm-Breschkin, J. L. Resistance of influenza viruses to neuraminidase inhibitors-a review. Antiviral Res. 47, 1-17 (2000).
5. Gubareva, L. V., Webster, R. G. \& Hayden, F. G. Comparison of the activities of zanamivir, oseltamivir, and RWJ-270201 against clinical isolates of influenza virus and neuraminidase inhibitor-resistant variants. Antimicrob. Agents Chemother. 45, 3403-3408 (2001).

6. de Jong, M. D. et al. Oseltamivir resistance during treatment of influenza A (H5N1) infection. N. Engl. J. Med. 353, 2667-2672 (2005)

7. Yen, H. L. et al. Neuraminidase inhibitor-resistant recombinant A/Vietnam/ 1203/04 (H5N1) influenza viruses retain their replication efficiency and pathogenicity in vitro and in vivo. J. Virol. 81, 12418-12426 (2007).

8. Lackenby, A. et al. Emergence of resistance to oseltamivir among influenza $A(H 1 N 1)$ viruses in Europe. Euro Surveill. 13 (2008).

9. Murphy, B. R. \& Webster, R. G. in Fields Virology 3rd edn (eds Fields, D. B. N., Knipe, M. \& Howley, P. M.) 1397-1445 (Lippincott-Raven, Philadelphia, 1996).

10. Varghese, J. N., Laver, W. G. \& Colman, P. M. Structure of the influenza virus glycoprotein antigen neuraminidase at $2.9 \AA$ resolution. Nature $303,35-40$ (1983).

11. Baker, A. T., Varghese, J. N., Laver, W. G., Air, G. M. \& Colman, P. M. Threedimensional structure of neuraminidase of subtype N9 from an avian influenza virus. Proteins 2, 111-117 (1987).

12. Burmeister, W. P., Henrissat, B., Bosso, C., Cusack, S. \& Ruigrok, R. W. Influenza B virus neuraminidase can synthesize its own inhibitor. Structure 1, 19-26 (1993).

13. Ives, J. et al. Anti-viral drug resistance: An oseltamivir treatment-selected influenza A/N2 virus with a R292K mutation in the neuraminidase gene has reduced infectivity in vivo. J. Clin. Virol. 18, 251-269 (2000).

14. Ives, J. A. L. et al. The H274Y mutation in the influenza A/H1N1 neuraminidase active site following oseltamivir phosphate treatment leave virus severely compromised both in vitro and in vivo. Antiviral Res. 55, 307-317 (2002).

15. Herlocher, M. L. et al. Influenza viruses resistant to the antiviral drug oseltamivir: transmission studies in ferrets. J. Infect. Dis. 190, 1627-1630 (2004).

16. Gubareva, L. V., Kaiser, L., Matrosovich, M. N., Soo-Hoo, Y. \& Hayden, F. G. Selection of influenza virus mutants in experimentally infected volunteers treated with oseltamivir. J. Infect. Dis. 183, 523-531 (2001).

17. Kiso, M. et al. Resistant influenza A viruses in children treated with oseltamivir: descriptive study. Lancet 364, 759-765 (2004).

18. Le, Q. M. et al. Avian flu: isolation of drug-resistant H5N1 virus. Nature 437, 1108 (2005).

19. McKimm-Breschkin, J. L., Selleck, P. W., Usman, T. B. \& Johnson, M. A. Reduced sensitivity of influenza A to oseltamivir. Emerg. Infect. Dis. 13, 1354-1357 (2007).

20. Rameix-Welti, M. A. et al. Natural variation can significantly alter the sensitivity of influenza A (H5N1) viruses to oseltamivir. Antimicrob. Agents Chemother. 50, 3809-3815 (2006).

21. Wang, M. Z., Tai, C. Y. \& Mendel, D. B. Mechanism by which mutations at His274 alter sensitivity of influenza a virus N1 neuraminidase to oseltamivir carboxylate and zanamivir. Antimicrob. Agents Chemother. 46, 3809-3816 (2002).

22. Yen, H. L. et al. Neuraminidase inhibitor-resistant influenza viruses may differ substantially in fitness and transmissibility. Antimicrob. Agents Chemother. 49, 4075-4084 (2005)

23. Smith, B. J. et al. Structural studies of the resistance of influenza virus neuramindase to inhibitors. J. Med. Chem. 45, 2207-2212 (2002).

24. Varghese, J. N., McKimm-Breschkin, J. L., Caldwell, J. B., Kortt, A. A. \& Colman, P. $M$. The structure of the complex between influenza virus neuraminidase and sialic acid, the viral receptor. Proteins 14, 327-332 (1992).

25. Russell, R. J. et al. The structure of $\mathrm{H} 5 \mathrm{~N} 1$ avian influenza neuraminidase suggests new opportunities for drug design. Nature 443, 45-49 (2006).

26. Cheung, C. L. et al. Distribution of amantadine-resistant H5N1 avian influenza variants in Asia. J. Infect. Dis. 193, 1626-1629 (2006).

27. Gulick, R. M. et al. Treatment with indinavir, zidovudine, and lamivudine in adults with human immunodeficiency virus infection and prior antiretroviral therapy. N. Engl. J. Med. 337, 734-739 (1997).

28. De Clercq, E. The design of drugs for HIV and HCV. Nature Rev. Drug Discov. 6 1001-1018 (2007).

29. Ha, Y., Stevens, D. J., Skehel, J. J. \& Wiley, D. C. X-ray structures of H5 avian and H9 swine influenza virus hemagglutinins bound to avian and human receptor analogs. Proc. Natl Acad. Sci. USA 98, 11181-11186 (2001).

30. Tai, C. Y. et al. Characterization of human influenza virus variants selected in vitro in the presence of the neuraminidase inhibitor GS 4071. Antimicrob. Agents Chemother. 42, 3234-3241 (1998).

Supplementary Information is linked to the online version of the paper at www.nature.com/nature.

Acknowledgements Work at NIMR was funded by the Medical Research Council (UK). This work was also supported in part by the EU FP6 Programme VIRGIL, contract number 503359. R.J.R. thanks the Scottish Funding Council for financial support.

Author Information Structural data have been deposited with the Protein Data Bank with accession codes 3CLO (His274Tyr-oseltamivir), 3CKZ (His274Tyr-zanamivir) and 3CL2 (Asn294Ser-oseltamivir). Reprints and permissions information is available at www.nature.com/reprints. Correspondence and requests for materials should be addressed to S.J.G. (sgambli@nimr.mrc.ac.uk). 


\section{METHODS}

Neuraminidase activity measurements. Michaelis-Menten constants $\left(K_{\mathrm{m}}\right)$ were determined using standard initial rate measurements with NA concentrations in the range 0.1 to $0.5 \mathrm{nM}$ and MUNANA concentrations in the range 2 to $200 \mu \mathrm{M}$. Dissociation constants for enzyme-inhibitor complexes were determined by measuring the reduction in the rate of MUNANA hydrolysis observed in the presence of different concentrations of the inhibitors. The $K_{\mathrm{I}}$ values were determined using equation (1) (Supplementary Information) for data collected at two or more different MUNANA concentrations and three different drug concentrations. The inhibitor concentration was always at least tenfold higher than the NA concentration to allow the approximation $[I]=\left[I_{\text {total }}\right]$.

The kinetic parameters for the inhibitors were measured in two ways: by adding enzyme to a pre-warmed mixture of MUNANA and inhibitor, or by adding inhibitor to a standard reaction mixture of enzyme and MUNANA approximately $50-200 \mathrm{~s}$ after initiation of the reaction. The two approaches gave identical results. In both cases, the exponential approach to the new steady-state rate was analysed using equation (2) (Supplementary Information). Kinetic measurements were made at two or more different MUNANA concentrations and three different drug concentrations. Association $\left(k_{\text {on }}\right)$ and dissociation $\left(k_{\text {off }}\right)$ rate constants for inhibitor binding were then determined using equation (3) (Supplementary Information).

Crystals were obtained by the vapour diffusion method from sitting drops dispensed with an Oryx 8 robot (Douglas Instruments). The drops consisted of $100 \mathrm{nl}$ protein $\left(6 \mathrm{mg} \mathrm{ml}^{-1}\right)$ with $1 \mathrm{mM}$ inhibitor (oseltamivir or zanamivir) mixed with $100 \mathrm{nl}$ reservoir solution of 15\% PEG 3350, 0.1 M sodium acetate $\mathrm{pH} 4.6$. Crystals were transferred into a cryoprotectant consisting of reservoir solution augmented with $20 \%$ (v/v) PEG 400 and inhibitor before flash freezing.

Data sets were recorded on a Raxis4 detector $(100 \mu \mathrm{m}$ scan $)$ mounted on a Rigaku MicroMax 007 HF generator and for Asn294Ser on the MARCCD at Daresbury station 10.1. Diffraction data were integrated using Denzo and scaled with Scalepack ${ }^{31}$. N1 mutant structures were solved by molecular replacement using AmoRe with the wild-type structure (Protein Data Bank code 2HU4) as the initial search model. Standard refinement was carried out with refmac5 (ref. 32) for the two His274Tyr complexes, and PHENIX ${ }^{33}$ for the Asn294Ser complex where non-crystallographic symmetry restraints were used. Manual model building was with $\mathrm{O}^{34}$ and $\mathrm{Coot}^{35}$. Figure 2 was created with Pymol (http:// pymol.sourceforge.net/).

31. Otwinowski, Z. \& Minor, W. in Data Collection and Processing (eds Sawyer, L., Isaacs, N. \& Bailey, S.) 556-562 (SERC Daresbury Laboratory, Warrington, 1993).

32. Collaborative Computational Project, Number 4. The CCP4 suite: programs for protein crystallography. Acta Crystallogr. D 50, 760-763 (1994).

33. Adams, P. D. et al. PHENIX: building new software for automated crystallographic structure determination. Acta Crystallogr. D 58, 1948-1954 (2002).

34. Jones, T. A., Zhou, J. Y., Cowan, S. W. \& Kjeldgaard, M. Improved methods for building protein models in electron density maps and the location of errors in these models. Acta Crystallogr. A 47, 110-119 (1991).

35. Emsley, P. \& Cowtan, K. Coot: model-building tools for molecular graphics. Acta Crystallogr. D 60, 2126-2132 (2004). 